\title{
Esophageal Lymphoepithelioma-Like Carcinoma with Unique Daisy-Like Appearance
}

\author{
Sehmus Olmez ${ }^{1}$, Alper Cann $^{2}$, Alpaslan Yavuz ${ }^{3}$, Umit Haluk Iliklerden ${ }^{4}$ and Gulay Bulut ${ }^{5}$ \\ Departments of ${ }^{1}$ Gastroenterology, ${ }^{2}$ Medical Oncology, ${ }^{3}$ Radiology, ${ }^{4}$ Surgery, and ${ }^{5}$ Pathology, Yuzuncu Yil University Medical Faculty, Van, \\ Turkey
}

Due to differences in prognosis and management, it is important to subclassify esophageal carcinoma. Esophageal lymphoepitheliomalike carcinoma (LELC) is extremely rare, with only a few cases reported to date. Review of the literature revealed case reports describing lesions with similar histology. We present a 69-year-old man with a giant pedunculated-polypoid lesion of the esophagus shrinking the lumen. Endoscopic excision of the tumor was performed and final histopathological diagnosis was confirmed to be LELC. In contrast to a previous case with a more aggressive course and a recurrent lesion, our patient died of his disease within 8 months of diagnosis. Here we discuss the endoscopic and radiologic findings of the case and a review of the literature. Clin Endosc 2015;48:549-552

Key Words: Lymphoepithelioma-like carcinoma; Esophagus; Prognosis

\section{INTRODUCTION}

Tumors of the esophagus other than squamous cell carcinoma and adenocarcinoma are quite rare. Lymphoepithelioma-like carcinoma (LELC) has been described as an unor poorly-differentiated form of squamous cell carcinoma associated with reactive lymphoplasmacytic infiltration. ${ }^{1}$ The stomach is the most common site for gastrointestinal LELC; however, esophageal involvement is occasionally observed. ${ }^{2}$ Esophageal LELC are primarily submucosal lesions with normal-appearing epithelial coverage and rarely can have polypoid, ulcerative, or reddish mucosal irregularity.

Herein, we present a case of this rare LELC. However, unlike a previous report, this patient's tumor was clinically aggressive, with radiographic evidence of metastatic spread, and the patient died 8 months after diagnosis. In addition, we

Received: November 9, 2014 Accepted: February 18, 2015

Correspondence: Sehmus Olmez

Department of Gastroenterology, Yuzuncu Yil University Medical Faculty, Van, Turkey

Tel: +90-432-216-7325, Fax: +90-432-216-7325, E-mail: drsehmusolmez@gmail.com

(c) This is an Open Access article distributed under the terms of the Creative Commons Attribution Non-Commercial License (http://creativecommons.org/ licenses/by-nc/3.0) which permits unrestricted non-commercial use, distribution, and reproduction in any medium, provided the original work is properly cited. demonstrate a case of esophageal LELC with combined morphology of reddish-white color, polypoid tumor, and mucosal irregularity.

\section{CASE REPORT}

The patient was a 69-year-old man seeking treatment for unintended weight loss, loss of appetite, and dysphagia. Routine blood laboratory test results indicated no abnormality. Thoracic computed tomography (CT) examination with intravenous contrast agent injection revealed a nodular, esophageal soft tissue mass at the level of the carina. The size of the lesion was $13 \times 10 \mathrm{~mm}$ axially (Fig. 1A). Paratracheal, subcarinal, and bilateral hilar lymphadenopathies were detected in the mediastinal region. Upper endoscopy revealed a giant pedunculated-polypoid lesion partially shrinking the esophageal lumen at $23 \mathrm{~cm}$ from the incisor. Yellowish-white exudative secretion over the polypoid lesion was present. The esophageal mucosa was irregular, reddish colored, and contained erosion from the level of the lesion to the cardioesophageal junction. The combination of polypoid tumor and mucosal changes constituted the unique endoscopic "daisy-like" figure (Fig. 2A, B). Sclerotherapy (adrenalin $4 \mathrm{~mL}$ 1/10,000) was administered in 

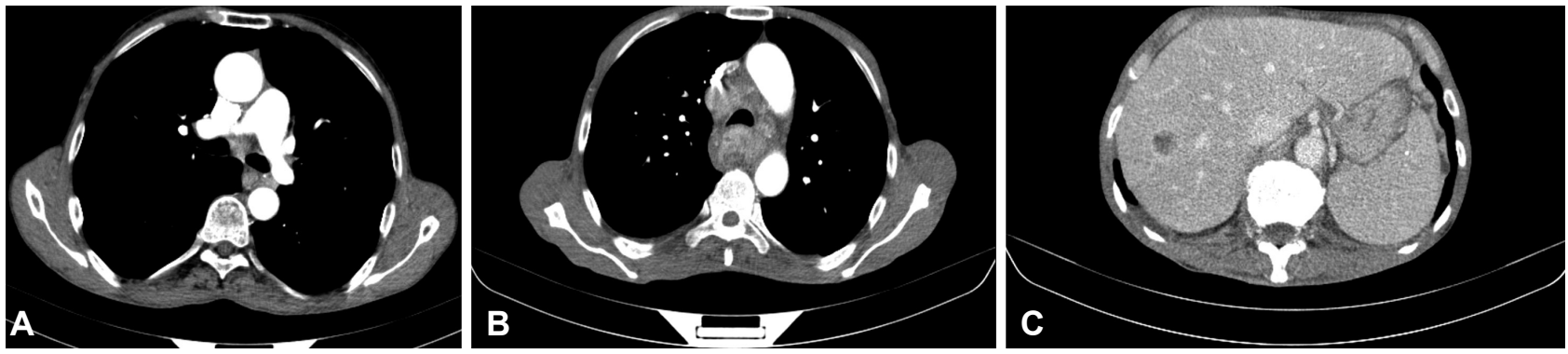

Fig. 1. Thoracic computed tomography (CT) scan with intravenous contrast medium administration. (A) Examination revealed nodular soft tissue lesion narrowing the esophageal lumen at the level of the carina. The size of the lesion measured $13 \times 10 \mathrm{~mm}$ in diameter. (B) Enlargement of the lesion was detected and mediastinal lymphadenopathies were established at 6-month follow-up on thoracic CT scan. (C) Abdominal CT imaging revealed liver metastasis.
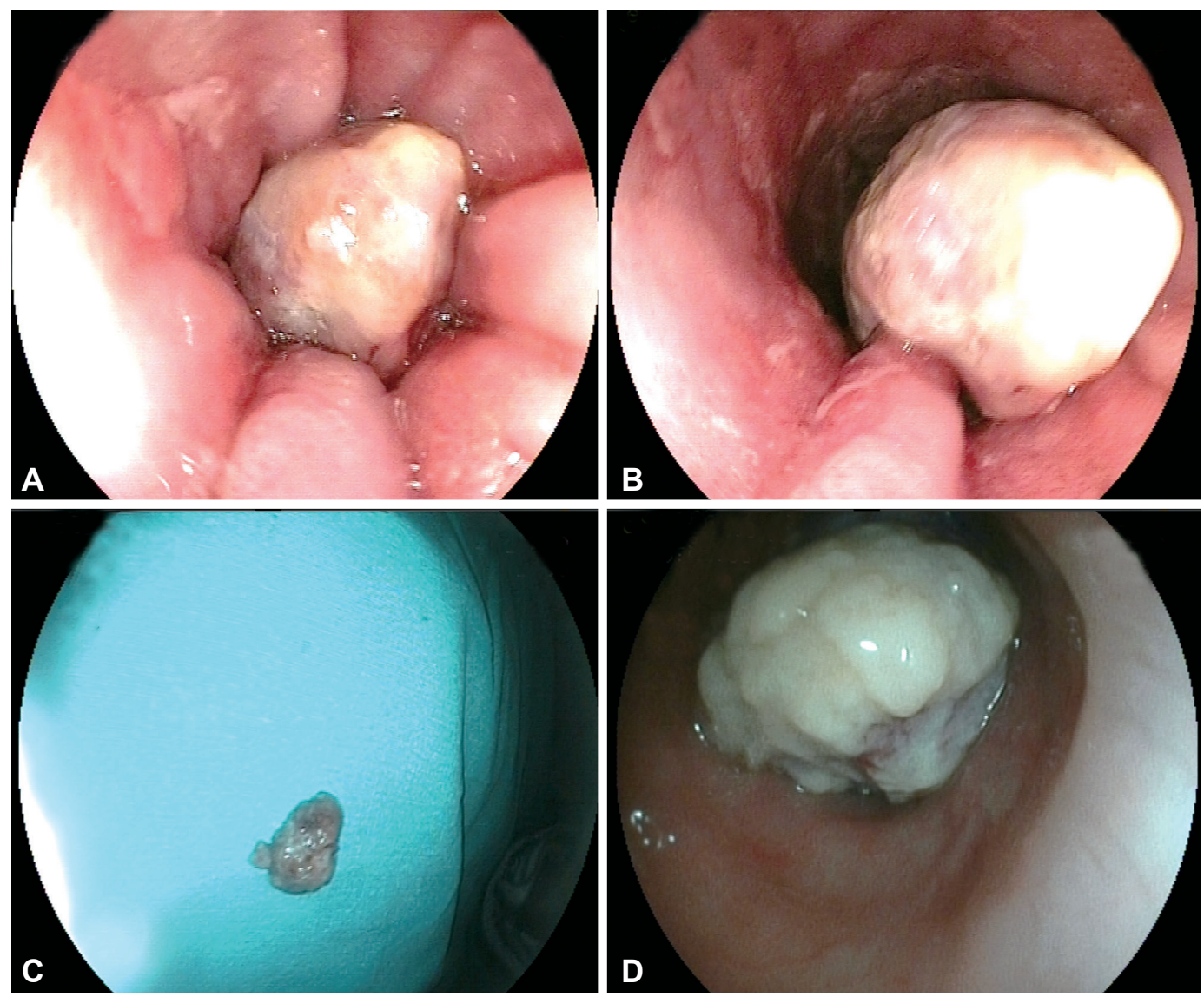

Fig. 2. Upper endoscopic examination. (A) Endoscopy revealed a giant peduncuated polypoid lesion partially shrinking the esophageal lumen at the level of $23 \mathrm{~cm}$ from the incisor. Yellowish-white exudative secretion over the polypoid lesion was determined. The combination of polypoid tumor and mucosal changes constituted the unique endoscopic "daisy-like" appearance. (B) Esophageal mucosa was irregular, reddish colored, and contained erosion from the level of the lesion to the cardioesophageal junction. (C) Endoscopic excision polypectomy was performed with snare. (D) Endoscopic examination revealed a giant pedunculated-polypoid lesion totally obliterating the esophageal lumen at the level of $24 \mathrm{~cm}$ from the incisor. The lesion surface was ulcerative and had a white exudative feature.

the base of the polypoid lesion, and endoscopic excision-polypectomy was performed with a snare (Fig. 2C).

Undifferentiated cells with large nuclei and poorly defined cytoplasmic borders, imparting a syncytial appearance, were determined by histopathologic observation. The background consisted of a prominent lymphoid infiltrate (H\&E stain, $\times 200$ ), and strong cytoplasmic staining with cytokeratin was determined (CK stain, $\times 200)$. CD20, CD3, synaptophysin, chromogranin, and p16 immunohistochemistries were all negative. CK and CK7 were positive while high-molecular weight cytokeratin and p63 were focal positive. Finally, the histopathological diagnosis was LELC (Fig. 3). The patient's complaint of dysphagia ended immediately after polypectomy. The patient did not accept the surgery. The patient was uncooperative for follow-up, and his re-consultation at our clinic was due to recurrence of aforementioned complaints 

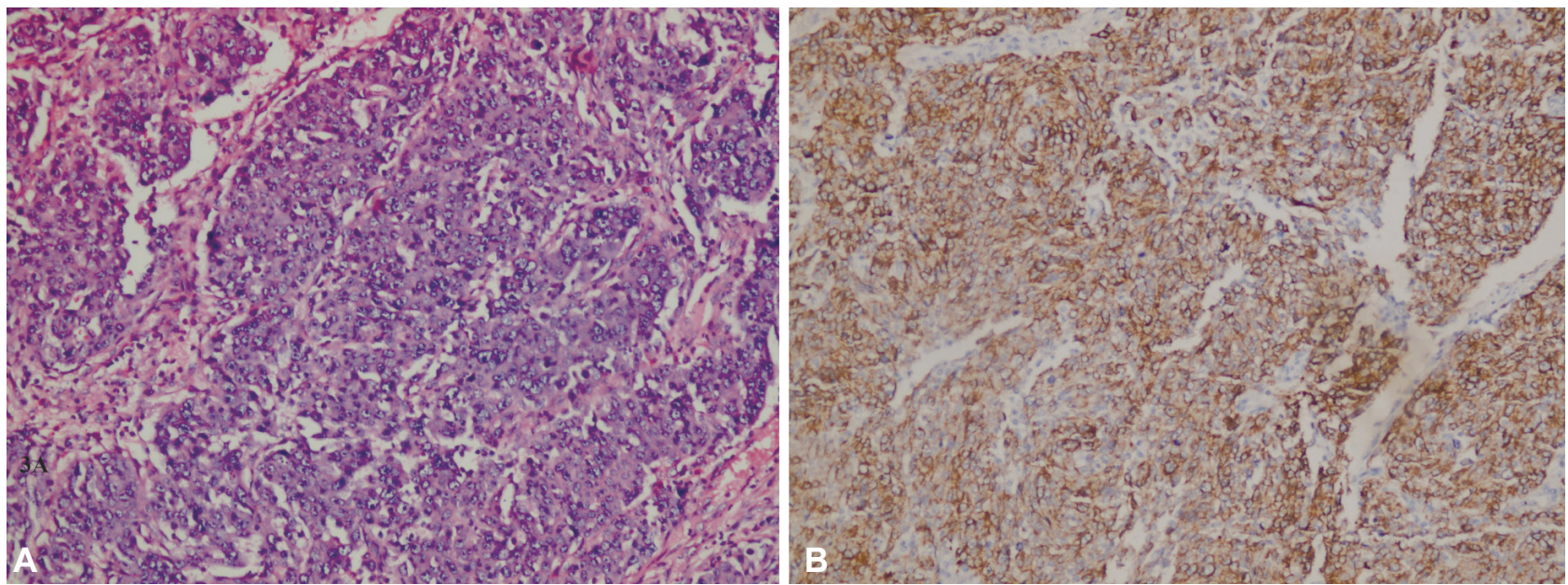

Fig. 3. Histopathologic examination of the lesion. (A) The tumor is characterized by sheets of undifferentiated cells with large nuclei and poorly defined cytoplasmic borders, imparting a syncytial appearance (H\&E stain, $\times 200$ ). (B) Strong cytoplasmic staining with cytokeratin in lymphoepithelioma-like carcinoma (CK, $\times 200)$.

after 4 months from initial diagnosis, such as dysphagia and loss of weight. Thoracic and abdominal CT examination with venous contrast medium injection revealed a recurrence resembling esophageal tumor with extended size accompanied by mediastinal lymph node metastasis at the periesophageal and subcarinal regions, as well as liver metastasis. (Fig. 1B, C). After 6 months, endoscopic examination revealed a giant pedunculated polypoid lesion totally obliterating the esophageal lumen at the level of $24 \mathrm{~cm}$ from the incisor. The lesion surface contained ulcerative and white exudative features (Fig. 2D). Surgical gastrostomy was performed at the 7th month and the patient died 8 months after the initial diagnosis.

\section{DISCUSSION}

Lymphoepithelioma was first reported in 1921 and described as undifferentiated carcinoma with distinct lymphoplasmacytic infiltration originating in the nasopharyngeal region. ${ }^{6,7}$ LELC tumors can arise in a multitude of locations such as the thyroid and breast, as well as gastrointestinal sites such as the biliary system, stomach, and colon. While the histologic features are similar, clinical presentation, age, sex, stage at presentation, size, and overall survival vary dramatically between sites. ${ }^{8}$ LELC has a better prognosis compared to conventional gastrointestinal adenocarcinoma. ${ }^{9,10}$ Seventeen LELC cases arising in the esophagus have been reported in the English language literature. ${ }^{8}$

The most characteristic endoscopic appearance of esophageal LELC is submucosal tumor covered with intact or ulcerative esophageal mucosa and a depressed middle portion. Exceptional cases may have simple ulcerative morphology, polypoid lesion, or solely reddish mucosal irregularity. ${ }^{8}$ In our case, the lesion was polypoid and the accompanying reddish mucosal irregularity was established from the lesion to the level of the cardioesophageal junction. The endoscopic "daisy-like" appearance of the lesion as the result of combined morphology was considered to be a noteworthy feature of this case. Limited information exists in the literature regarding the long-term follow-up and management of LELC because of its low incidence. Nakasono et al..$^{8}$ reported a case of esophageal LELC with non-progressive behavior. Contrary to that report, our case had a rapid recurrence after polypectomy with concomitant evident lymphadenopathies, which can indicate poor prognosis. Surgery, radiotherapy, or chemotherapy can be treatment options depending on the location of the lesion, and better survival rates can be achieved with these treatments. This treatment protocol is also valid for esophageal LELC and may lead to a better prognosis. ${ }^{11}$

In conclusion, we present the radiologic and unique endoscopic features of an extremely rare esophageal LELC case. Polypoid morphology with mucosal irregularity and rapid progression after polypectomy were the striking findings of the case, which should be considered for differential diagnosis of lesions with similar features.

Conflicts of Interest

The authors have no financial conflicts of interest.

\section{REFERENCES}

1. Tsang WY, Kuo TT, Chan JK. Lymphoepithelial carcinoma. In: Barnes L, Eveson JW, Reichart P, eds. Pathology and Genetics of Head and Neck Tumours. Lyon: IARC Press; 2005; p. 251-252.

2. Gurzu S, Szentirmay Z, Bara T, et al. Non-Epstein-Barr virus associated lymphoepithelioma-like carcinoma of the esophagogastric junction with microsatellite instability, K-ras wild type. Pathol Res Pract 2013;209:128- 
131.

3. Angulo-Pernett F, Smythe WR. Primary lymphoepithelioma of the esophagus. Ann Thorac Surg 2003;76:603-605.

4. Kuo T, Hsueh C. Lymphoepithelioma-like salivary gland carcinoma in Taiwan: a clinicopathological study of nine cases demonstrating a strong association with Epstein-Barr virus. Histopathology 1997;31:75-82.

5. Tardío JC, Cristóbal E, Burgos F, Menárguez J. Absence of EBV genome in lymphoepithelioma-like carcinomas of the larynx. Histopathology 1997;30:126-128.

6. Regaud C, Reverchon L. Sur uncasd'epitheliome epidermoide developpe dans les massif maxillaire superieur. Rev Laryngol Otol Rhinol 1921;42:369-378

7. Schmincke A. Über lymphoepitheliale Geschwülste. Beitr Pathol Anat 1921;68:161-170.
8. Nakasono M, Hirokawa M, Suzuki M, et al. Lymphoepithelioma-like carcinoma of the esophagus: report of a case with non-progressive behavior. J Gastroenterol Hepatol 2007;22:2344-2347.

9. Falzarano SM, Mourmouras V, Mastrogiulio MG, La Magra C, Vindigni C. Undifferentiated gastric carcinoma with lymphoid stroma (lymphoepithelioma-like carcinoma/medullary carcinoma). Pathologica 2009;101:15-17.

10. Papalambros E, Felekouras E, Pikoulis E, et al. Epstein-Barr virus: associated adenocarcinoma of the stomach: a rare entity with distinct characteristics. J BUON 2003;8:329-331.

11. Sashiyama H, Nozawa A, Kimura M, et al. Case report: a case of lymphoepithelioma-like carcinoma of the oesophagus and review of the literature. J Gastroenterol Hepatol 1999;14:534-539. 